\title{
PERSONAL ONLINE IDENTITY-BRANDING OR IMPRESSION MANAGEMENT
}

\author{
Maria Magdalena POPESCU \\ popescu.maria@myunap.net \\ “CAROL I” NATIONAL DEFENSE UNIVERSITY, BUCHAREST, RoMANIA
}

\begin{abstract}
It is said that western society is heading toward individuation, towards personalization. This, along with continuous technological progress, triggers the concept of Personal Identity Online (PIO) which highlights and tailors a specific characteristic of an individual's behavior in an online network of similar ones, benefitting from a single opportunity to shape an individual's identity differently from the one he has in reality. Looked at from the network of ties existent in a social online environment, identity is tailored by each individual representation in virtual encounters. This representation is provided by the users' profiles while the posts used are enriched and shared account for the visual representation of alterity. The present paper looks at how impression management and personal branding are developed in the social network environment in a desire to complete personal characteristics that reality did not grant. Analysis on posted content, management of information and social manifestation are involved to this end.
\end{abstract}

\section{KEYWORDS:}

Branding, impression management, online identity, sociality

\section{Introduction}

Studying the online representation of individuals, more and more intelligence can be gathered, as proved by spreadsheets used by the recent Cambridge Analytica data retrieval of consumers' profiles in mundane transactions exposure, by human resources departments continuous head-hunt or by political and terrorist organizations profiling in search for newbies, due to the individual's online presence even when he or she is no longer logged on. Since one can send messages or can comment on someone else's post even though the target is no longer online, this triggers the fact that the interactions are performed among imagistic identities not among people, with dialogues performed among posts, images, quotes or statuses. In this respect, research questions here the reality factor in the general management of senses, since individuals exhibit a "general alterity" in a permanent exchange for impressions (Mead, 1936), a scaffold used to anticipate people's reactions based on the desired or tailored self-image attributes. Moreover, studies show that identities built are generally augmented, compensating for the lack of 
nonverbal communication or visual contact (Geidner, Flook \& Bell, 2007), a situation in which one strives more to be among the best people in the group (Buffardi \& Campbell, 2008) by posting or reacting to images or phrases that clearly show one's orientation and group (Strano, 2008; Young, 2008). It is said that all individuals online build their alternate personas for an environment meant for revealing themselves to others. Entirely New personae are presented to the public. Since the delineation between the online and offline are blurred by technology, the most intimate things about oneself are being affected significantly, thus self-perception also changes along with the person we build online, to induce to the other peers. However, a proper selection of words and posts communicates best (Siibak, 2009) due to the process of presentation, tagging, sharing, commenting or associating to other people or other posts, all of them part of the impression management (Strano, 2008) or personal branding (Young, 2008; Siibak, 2009).

\subsection{Definition of concepts}

Impression management as a term has been first conceptualized in 1959, to be later re-defined (Schlenker, 1980), as the "attempt to control images that are projected in real or imagined social interactions" (Schneider, 1981, pp. 23-40) pointed out that impressions may be managed by means other than selfpresentation. For example, impressions of an individual may be managed by a third party, but the process always comprises of two components, impression motivation and impression construction (Leary \& Kowalski, 1990). Personal branding on the other hand has often been approached in conjunction with impression management, as the active part of the planning process. In other words, "personal branding" is seen as the way people market themselves and their careers similar to the way companies manage their consumer brand.

In a search to render a more complete definition of concept, one would say that branding is everything that identifies a construct of reality from others similar to the one. As a continuation, personal branding is what makes people stand out as individuals to differentiate from all the other ones similar to them, it is the situation when "what do you want to be known for?" (Dalla-Camina, 2016) is a question to be asked, especially when inordinate numbers of social networking applications are conducive to building ourselves several images, different from one another, allowing one to communicate on various networks, in various ways (synchronous or asynchronous, live or text, image or sound). In contributing to the images one builds about oneself to develop a personal brand, it is not important what we do, but how we do it, for whom and why we do it. Creating a brand for oneself is establishing how one makes a difference in the world and highlights those differences. However, not projecting a carefully planned impression management and mixing aspects about oneself to let people know us for various reasons (Harris \& Rae, 2011) is dangerous, producing confusion. Social Networking Sites are the most popular and obviously the most practical medium conducive to developing one's online identity, to tailoring a reputation in order to become visible in the group that counts. By making a difference in the online environment, one can be consistent with oneself, in a planned posting and commenting itinerary from status to posts and reactions in order not to lose trust and gain acceptance and confidence. Bearing in mind that each social media application attracts a certain type of people (Kaplan \& Haenlein, 2010) (blogs attract opinionated individuals, Twitter is for the pragmatic ones, Facebook is more tailored to emotions and connection, while Instagram is the app for image people) we will undertake an analysis of the apps for these particular types of people, but we will consider that one needs to choose the right medium for 
the right social manifestation of the online identity. Even if one chooses to be active on more than one social network in order to ensure reach and, ultimately, to impress (where reach is the number of people who can see your content, they are exposed to it, while impression is the number of times your content is displayed, irrespective of the fact that it was accessed or not), consistency is highly important for a solidly contoured identity.

\section{Expressions of personal and social identity in social media}

Identity represents the expression of a person's individuality in the context of a person's affiliation to certain groups, the ability to differentiate oneself amidst the crowd. Psychologically speaking, identity depends on a multitude of elements like self-image (mental representation of a person about oneself), self-esteem and individuality. Sociologically speaking, identity is related to behavior, the result of manifested attitudes and social roles. If, psychologically speaking, identity refers to the uniqueness of the individual, sociologically speaking identity relates to the features one has identical to the group the person belongs to. Also, sociologically speaking, identity is already anchored in the relationship between the individual and the collective, between the people and the groups they belong to (Cooley, 1902). Both individually and socially, identity is seen as an alterity, as a contrast, a highlight of otherness that can be changed, improved or altered (Mead, 1936) due to various factors' influence. Personal identity, on the other hand, is the subjective side of one's complexity and manifests itself in organizing goals (needs, wishes) and abilities (competences, skills) in the context of cultural or personal demands and gratifications (Marcia, 1994). In the social context, people interact with one another and it is the social identity that manifests itself, it is that side of the individual that is responsible for changing attitudes in relation to changing factors in the social context one interacts with. Digitally, one can often speak about "different ego states of a person in transactions with another person's ego states" (Armean, 2018).

\subsection{Research questions}

Our analysis is meant to understand the Romanian online social users' built identity and how interactions among imagistic identities are performed, since the dialogue in social networking sites develops among posts, images, quotes or statuses instead of persons. We intend to see whether the identities built for interaction with others in the online are generally augmented, compensating for the lack of nonverbal communication or visual contact, a situation in which one strives more to be among the best people in the group by posting or reacting to images or phrases that clearly show one's orientation and group. For this, we will consider a proper selection of posts that communicate best due to the process of presentation, tagging, sharing, commenting or associating to other people or other posts, all of them part of the impression management or personal branding. Focusing on answering the question "what do you want to be known for?" (Strano, 2008) responsible for people's building several images, different from one another, with the goal of communicating on various networks, in various ways, we will seek to understand if there is a difference between personal and social identity and which of the two is the one responsible for the behavior one has in various appearances in social networks? How does one build and manage one's presence in social media in order to use it and meet his personal, social and cognitive gratifications? If personal identity is responsible for organizing goals and abilities to meet gratifications, then which is the social side of the identity that triggers changing attitudes? How do we count and consider various representations of the self in the online environment, as separate identities or as replicas of a unique one? 
These questions will be addressed in the upcoming analysis.

\subsection{Data and methods}

To answer these questions, we applied the qualitative method by extracting social media data from texts, images, first type connections i.e. friends and followers and second type connections, i.e. likes, comments and shares. We also extracted data from observing spontaneous interactions and mundane communication. In order to do this, we looked at events, opinions, feelings, network interactions and audience. The context of the analysis is Romania. Our sample counted top twenty blogs and the corresponding Facebook pages and their accounts. We then looked at twenty Facebook pages and profiles for each category of age, gender and educational background for the most active urban users.

Moreover, we resorted as well to the monitoring instruments used by specialized companies which have issued a digital media report coverage and among others, the internet usage, the type of online interactions and applications mostly used in Romania. Results show that the Internet use in urban areas is over $70 \%$ in most of the East European countries reported to daily usage, while the internet urban consumption in Romania is $80 \%$ of a population with an age range varying from 16 to 74 years. What is visible in the collected data is that all age groups have increased numerically in 2017, even though the segment 16-24 years old is seen as the most active, followed by the ones between 25 to 34 and then by the 35 to 44 years old.

Data registered considered the fact that Facebook provided the possibility for its users to avoid sensitive topics categories like social issue debates, tragedies, conflicts, dating and gambling, given the fact that Facebook has doubled its people who identify and remove content violations and fake accounts and announced that they are making efforts to fight fake news and click baits.
In this context, the analysis of the monitoring services providing data for Digital Media Report 2018 looked at the online activities people unfold, and they gathered data to profile the average user's online activity. There was a stable trend of search for information and online games, for 2017, a decrease in email use and an increase in social network usage and video watching. On average for Romania, Facebook was the leader which maintained the highest increase up to 9.8 mil users in the first semester of 2018 versus 8.4 at the end of 2017. This was followed by LinkedIn (which grew from 1.74 mil users in 2016 to 1.82 mil users in 2017), important numbers considering the app and its users have a prominent business perspective, then in line came Skype, Instagram and Twitter.

Beside all of the above, we used monitoring sources and individual analysis with a focus on the most highly ranked sites in terms of visibility and appreciation. By looking at statistics, figures confirmed Facebook as the leading application in the Romanian social media, with a total of reported subscribers of approximately 8.900.000 in December 2017 to grow in March 2018 to reach 9.900 .000 including both personal profiles and pages, (with a number of 83.575 pages from the given total) (pages are public version of the personal profile). On the other hand, figures for blogging in Romania show a number of 95.805 registered blogs out of which only 7.813 are active, approximately $8 \%$. This signifies a decline in interest as well as a selection and professionalization of the readers, an increase in quality and selection of trust from the readers' point of view. Conversely, the number of posts in blogging shows a figure of 101.336 with 97.559 comments, showing that there is at least one comment per blog posting. As a comparison, the interactions on Facebook reach 4.826 .314 , a figure that is half the number of the Facebook users, not to mention the number of posts on each user's timeline. The bloggers that have been 
accepted and recognized as solid and professionally endorsed opinion generators have gathered and built a public for whom posts grow constantly, bringing comments and subsequently user generated content in its turn. However, many of the Facebook accounts are abandoned and closed while the blogging account, though in a much smaller number, stay alive by constant number of users, comments and interested parties, ensuring a long term visibility of the blogs, even though it seems that while the use of Facebook raised, the use of Blogging nearly stalled, with the number of posts in blogging dropping. If we looked at Facebook users only, statistics showed that most of the users are from the urban areas, with a high-school degree $38.1 \%$ and higher education $37.3 \%$ with the rest in vocational and elementary school education background, in a balanced gender percentage.

Given the figures that indicate an uprise in social media use in urban Romania especially in Facebook and given the replication of accounts on various apps seen in Romanian users corroborated with approaches on uses and gratifications theories related to ways to communicate online that complement the displayed attitudes in the online environment, the research question here is how do the Romanian social media users manifest online and how do they build their social presence?

Considering the above mentioned data and the conclusions triggered, after having corroborated the blog author's account with his Facebook and other similar social media pages he runs, in order to identify similarities and differences, we chose blogs and Facebook pages to be compared.

We observed that the most visible and highly considered blogs (based on content criteria) were the ones on cooking and food, with slight variations, on IT, entertainment, life-style opinions and general advice. However, should we take the source that categorizes blogs based on authors, the ranking differs from one to twenty (Zelist Monitor; Biz Magazine). We selected the second source and categorization, authorbased, to perform analysis on the blog content. We then related the first type data to the new data extracted from the same authors' Facebook or other social media page. We corroborated information and drew conclusions on content management and attitude.

\subsection{Results and discussions}

The results that we obtained subsequent to the analysis we performed show us that social online identity is more important for men compared to women (65\% of the users of blogging in the most highly ranked contents are men, with the rest of $35 \%$ women) with an average age of 30 to 45 . Furthermore, the analyzed categories of account users split, with some using social media to build an extension of their own personality, a social alterity based on the background and the context they perform daily tasks in, based on the professional affiliations they have, and we shall call them the multiphrenia performers, while the rest of the users in focus totally reinventing themselves by means of social network, to manifest themselves in a desired and targeted world, conducive to a new identity, and we shall call these the personal branding performers. The latter ones strive for performing personal branding and impression management in order to advance into the online limelight.

For the subjects working in a certain field (i.e. media, technology, PR, etc.), the content comes easily at hand, since all is a promotion of information or events, a translation of their daily activity into the virtual environment, with a focus on their own professional achievement. For the ones reinventing themselves it is a planned impression management to get a contoured personality in a desired field, to become the online would-be professional. The ratio of consensus between the real and online identity is $60 \%$ to $40 \%$ in favor of the 
people who only extend their socioprofessional identity to an online alterity, similar to what is exposed in the real environments.

\subsection{The interpretation of results and discussion}

The replication and dissipation of online identity which some defined as multiphrenia is performed by all of our subjects, in order to build strong ties in the virtual environment. The content developed on other pages or inside other social networks aims only to repeat the same content exposed on the blog, in order for more visibility and more ties, for more followers or for more appreciation (likes). People who do personal branding are especially the ones reinventing themselves in the online environment (examples of former engineers acting as media people in the online environment, former social workers acting as event planners online, etc.). It is self-explanatory why those extending their social and professional identity online (with very slight variations) do not perform personal branding - it is the real professional context that acts as such for them.

Facebook accounts are used both as "people page" and as "public page". Sometimes, the people page exposes snapshots of personal identity in images and comments, while the public page exposes posts speaking of the would-be online-built socio-professional identity or shares and comments related to the professionally exposed profile. Images are a strong content that backs-up all the comments and opinions initiated on Facebook and blogs, with the aim to develop, perform and highlight the online social identity. Personal information is opaque for those who perform online alterity, it is only the social identity that is exposed, with details that are consistent to the real professional content, related to professional status. The ones performing personal branding are highly self-esteemed, they ask for support and are very careful in re-distributing content that has not been exposed too much. These are the ones that appeal to Vlogging as well, in a planned desire to persuade and penetrate the new environment where they tailor themselves into "the new me". Also, those that perform personal branding expose themselves personally slightly more - they post photos with family and friends, they give their background and demographics, in a manifestation of lust for trust. Related to gaining trust, all user accounts have real profile pictures. Attitude displayed is objective in posting images or travel videos, while it is subjective for comments, posts or shares for both categories, the multiphrenia performers and the personal branding performers.

With respect to architecture of ties, the results of the analysis for the networking sites in focus expose weak ties. Initial posts are frequent but words exchanged on the walls vary from few to many, based on the topic they advance. Usually politics generates comments which cannot bring intensity on ties as political topics are not constant in attitude or opinions. As far as the number of friends is concerned, the study noticed that even though the figures are very high (from 2000 to nearly 5000), there are shallow ties since the frequency and number of comments and shares do not exceed $10 \%$ from the number of appreciations each post gets and despite the fact that time lapse between comments and posts is very low (at most one or two hours). Even though the intimacy is low with weak ties, reciprocal favors are frequent in the form of common groups, endorsements and links exchanged or comments showing emotional support. As far as the strength of ties is concerned, the study has revealed that this is built by social presence on more than two social networking sites, the same identity and the same postings, but enlarged communities. We should draw attention here on the hybridization of ties, since the strength of ties built this way is actually weak, since ties are built with acquaintances, with 
people whose social and spatial proximity may turn each individual into a temporary half-reliable tie.

In the charts presented below, one can see the content topics used in the process of building a social identity (Figure no. 1) for the cases we studied along with the social networking sites favored by gender in building a social identity (Figure no. 2).

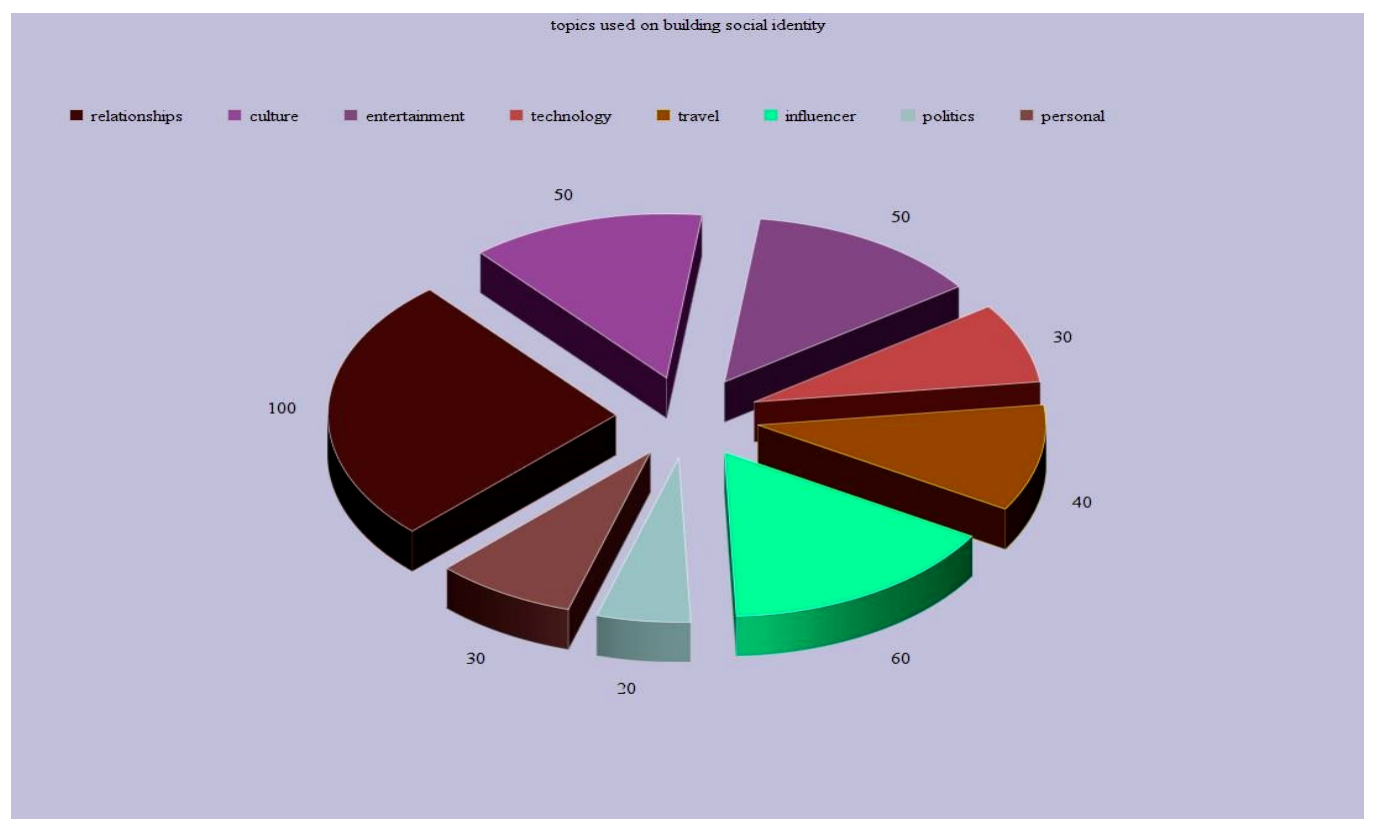

Figure no. 1. Content topics in building a social identity

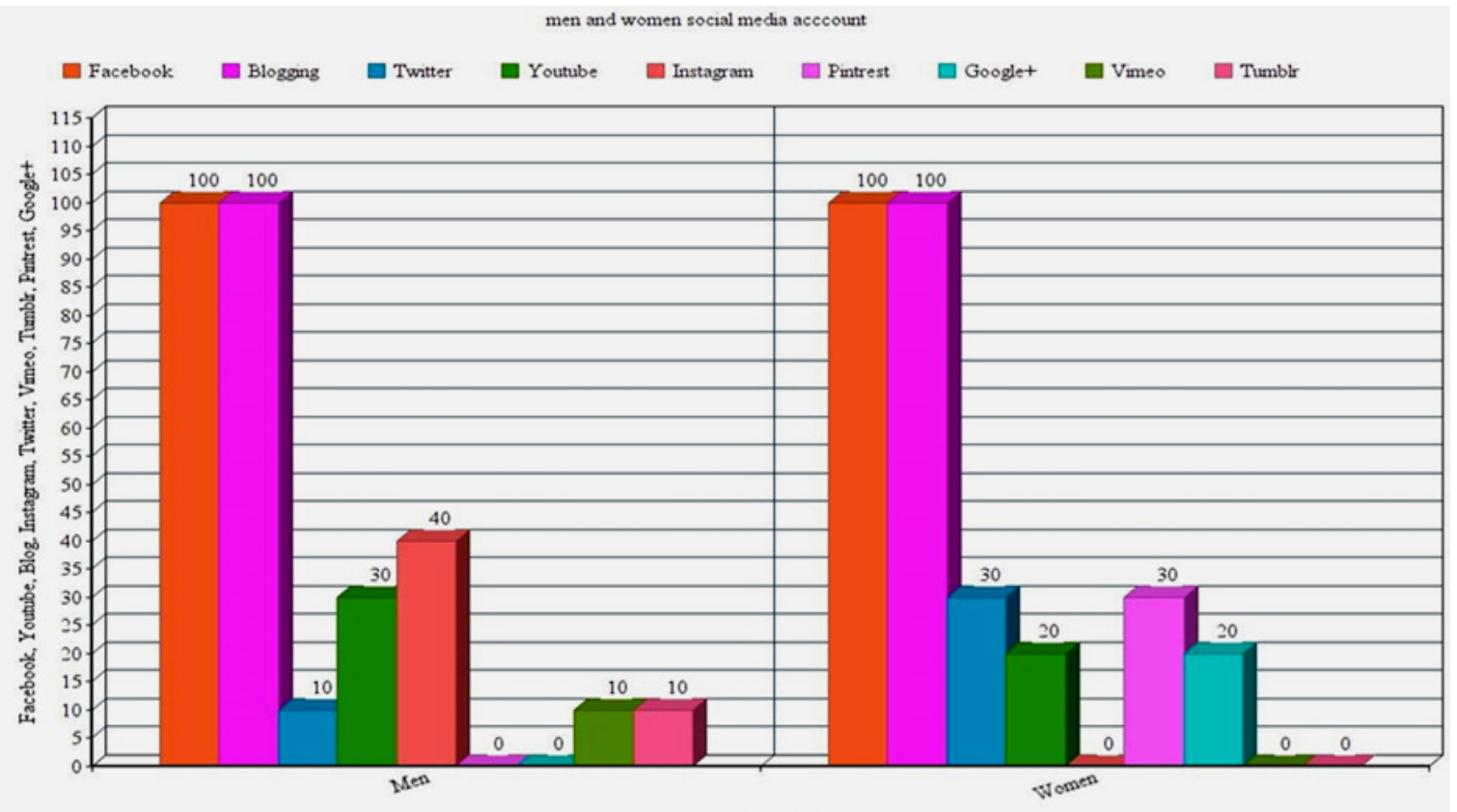

men, women

Figure no. 2. Gender-differentiated social media account 


\section{Conclusions}

The present paper looked at how and why communication develops to sustain identity in social networking sites, at how the ties are developed among social interactions when dialogue is performed and how identity is shaped or changed out of or along with these interactions. We questioned ourselves whether people shape their identity to please others or it is an inherent change brought along by the production of communication and feedback, by the dialogic interactions. To do all this we analyzed FB accounts, personal ones and pages and compared the information with analysis performed on blogs and Instagram. From the analysis we concluded the following: imagistic identities show an augmented reality of the characters created online either through angles of snapshot experiences or from qualifiers connected to the posts, highlighting embedded positive characteristics or sending positive messages about the snapshots presented in the posting. Posts that define the tailored identity are endorsed or highlighted either by a short key word description or by tagging popular names to connect with, nouns that have a certain significance for the highlighted quality/person/object/ experience. As far as content is regarded, the observed posts are advancing either a context or realia or events that speak for themselves, with an embedded significance of authorship for the one who posts.

As far as the content on blogs is concerned along with identity, we concluded that: most of them are a continuation of their work and activity, in the same field. The ones who reinvent themselves change direction and come from media or medical field for example and they promote ideas and product from IT or lifestyle. Many of them share other postings of interest in order to stay in their visitors' attention, or comment upon mundane events.
As far as the number of friends is concerned in building an identity, the study noticed that very high figures (from 2000 to nearly 5000) often lead to shallow ties, an unexpected turn, since the frequency and number of comments and shares do not exceed $10 \%$ from the number of appreciations each post gets, despite the fact that time lapse between comments and posts is very low (at most one or two hours). Another unexpected aspect is that even though the intimacy is low with weak ties, reciprocal favors are frequent in the form of common groups, endorsements and links exchanged or comments showing emotional support. In relation to the strength of ties, the study has revealed that this is built by social presence on more than two social networking sites, the same identity and the same postings come up on at least two different application accounts, with enlarged communities. These aspects draw attention on the hybridization of ties, since the strength of ties built this way is actually weak, due to the fact that ties are built with acquaintances, with people whose social and spatial proximity may turn each individual into a temporary halfreliable tie any moment in time.

In a nutshell, the study revealed the fluidity of online behavior and the hybrid state of ties built within online communities, as well as the complex structure in the architecture of ties. Personal branding is performed by people who reinvent themselves socially and professionally online in order to obtain acceptance and validate their newly acquired status, posting as a newly different professional, while the professionals who perform multiphrenia do that to adapt their own professional and social identity, not changing the general lines of social conduct, to tailor to various environments yet as a continuation of the real medium. To this end, personal identity coexists with social identity in a fluid continuation between the online and offline. 


\section{REFERENCES}

Armean, O. (2018). Skills of skepticism and judgement or how to be digitally adult. Bulletin of the Transilvania University of Braşov, Special Issue Series VII: Social Sciences and Law, Vol. 11 (60), No. 1, 11-16.

Biz Magazine, available at: https://www.revistabiz.ro/smsbucuresti2018/digital-report/, accessed on 08 April 2019.

Buffardi, L. E., \& Campbell, W. K. (2008). Narcissism and social networking websites. Personality and Social Psychology Bulletin, York University.

Cooley, C. H. (1902). Human nature and the social order. New York, USA: Charles Scribner's sons.

Dalla-Camina, M. (2016). What do you want to be known for?, available at: www.huffingtonpost.com.

Geidner, N. W., Flook, C. A., \& Bell, M. W. (2007). Masculinity and online social networks. Male self-identity on Facebook.com. Easter Communication Association $98^{\text {th }}$ Annual Meeting, Providence, RI.

Harris, L., \& Rae, A. (2011). Building a social brand through social networking. Journal of Business Strategy, Vol. 32, Issue 5, 14-21.

Kaplan, A. M., \& Haenlein, M. (2010). Users of the world unite! The challenges and opportunities of Social Media. Business Horizons, Vol. 53, Issue 1, 59-68.

Leary, M. R., \& Kowalski, R. M. (1990). Impression Management: A Literature Review and Two-Component Model. Psychological Bulletin, Vol. 107, Issue 1, 34-47, available at: http://dx.doi.org/10.1037/0033-2909.107.1.34.

Marcia, J. E. (1994). Ego identity and object relations. In Masling, J. M., \& Bornstein, R. F. (Eds.), Empirical perspectives on object relations theory (pp. 59-103). WA, USA: American Psychological Association.

Mead, G.H. (1936). Movements of Thought in the $19^{\text {th }}$ Century. Edited by Marrit H. Moore, Chicago, Illinois, USA: University of Chicago Press.

Schlenker, B. R. (1980). Impression Management: The Self-Concept, Social Identity, and Interpersonal Relations. Monterey, California, USA: Brooks/Cole Publishing Company.

Schneider, D. J. (1981). Tactical self-presentations: Toward a broader conception. In Tedeschi J. T. (Ed.), Impression management theory and social psychological research (pp. 23-40). New York, USA: Academic Press.

Siibak, A. (2009). Constructing the self through the photo selection-visual impression management on social networking websites. Cyberpsychology: Journal of Psychosocial Research on Cyberspace, Vol. 3, No. 1.

Strano, M. M. (2008). User descriptions and interpretations of self-presentation through FB profile images. Cyberpsychology: Journal of Psychosocial Research on Cyberspace, Vol. 2, No. 2.

Young, K. A. (2008). Online social networking: an Australian perspective. AOIR 0.9 Conference, Copenhagen, Denmark.

Zelist Monitor, available at: https://www.zelist.ro/bloguri/2018-03-13, accessed on 08 April 2019. 